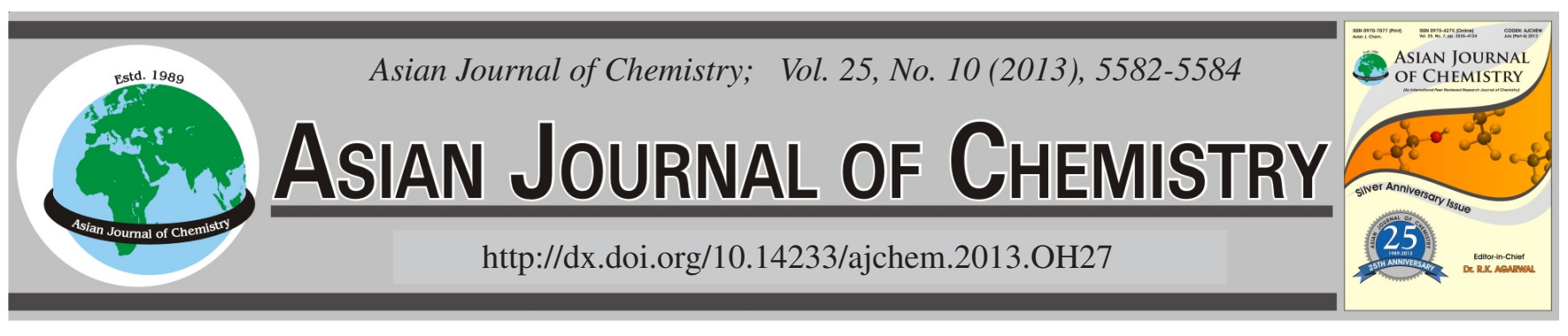

\title{
Study on the Bioactive Properties of Polyetheretherketone $\uparrow$
}

\author{
Yusong Pan ${ }^{*}$, Chuyang Xu and Chengling Pan
}

Department of Material Science and Engineering, Anhui University of Science and Technology, Huainan 232001, P.R. China

*Corresponding author: E-mail: yusongpan@163.com

\begin{abstract}
Polyetheretherketone has been increasingly employed as biomaterials due to its excellent biological characteristics. In this paper, the bioactivity of polyetheretherketone was investigated. The results showed that the bioactivity of polyetheretherketone could be improved by various techniques such as plasma treatment, surface grafting, surface deposition and addition of bioactive glass ceramic in the polyetheretherketone matrix. The aim of plasma treatment is to change the surface physicochemical properties of polyetheretherketone, thus enhancement the surface bioactivity of polyetheretherketone composites. Surface coating technique is devoted to improve the bioactivity of polyetheretherketone by deposition of bioactive ceramics such as hydroxyapatite and calcium phosphate on the surface of polyetheretherketone. Surface grafting modification is mainly attributed to improve the surface hydrophilicity and wettability through the grafting with hydrophilic or specific functional groups on the materials surface, thus enhancing bioactive performance of the material. The advantages of compounding bioactive glass ceramic with polyetheretherketone matrix are not only improving the bioactive properties but also increasing the mechanical properties of polyetheretherketone.
\end{abstract}

Key Words: Polyetheretherketone, Bioactivity, Surface modification, Biocomposites.

\section{INTRODUCTION}

Polyetheretherketone is a semi-crystalline thermoplastic, which has sufficient strength and stiffness with longterm biocompatibility. Due to its similar mechanical properties to that of natural bone and excellent biocompatibility, polyetheretherketone is used in a wide range of medical applications such as orthopedic, bone plate and spinal implants. Compared with traditional implants such as stainless steel, $\mathrm{Co}-\mathrm{Cr}-\mathrm{Mo}$ and $\mathrm{Ti}_{6} \mathrm{Al}_{4} \mathrm{~V}$ alloy, the modulus of polyetheretherketone is closer to that of natural bone ${ }^{1}$. These excellent performances could effectively reduce stress shielding due to the match in modulus of elasticity and stiffness between implant and bone. However, a major difficulty encountered with polyetheretherketone implants is to achieve a secure attachment between the implant and the surrounding bone because of its deficient bioactivity. For improving the biocompatibility of polyetheretherketone as an orthopedic implant, various studies have focused upon the bioactive properties of the polymer, either via adding bio-ceramic agents or surface modification ${ }^{2,3}$.

In this paper, the surface modification technologies which used to improve the bioactive properties of polyetheretherketone were investigated.
Surface modification: Surface modification is mainly to change the surface physical and chemical properties of the materials, such as the improvement of wettability and reinforcement of cell adhesion capacity on the materials surface, so as to achieve the aim of improving surface bioactivity. The technologies used to improve the bioactivity of polyetheretherketone based composites are mainly focused on the four aspects such as plasma treatment, surface grafting, surface coating and addition of bio-ceramics in polyetheretherketone matrix.

Plasma treatment: Plasma treatment technology is that the modified materials are placed in a specific environment to receive plasma irradiation or ion implantation. The surface physical and chemical properties such as wettability or surface hardness then would be changed due to the plasma irradiation or ion implantation.

Ha et al. ${ }^{4}$ investigated the polyetheretherketone surface bioactivity through plasma activation treatment in a $\mathrm{N}_{2} / \mathrm{O}_{2}$ plasma environment. It was observed that plasma treatment environment had a significant effect on the wettability of the polyetheretherketone surface. The bioactivity experiment results showed that plasma activation followed by hydroxyapatite nucleation in a supersaturated calcium phosphate solution had a positive effect on the growth rate of the layer on polyetheretherketone. McKenzie et al. ${ }^{5}$ investigated the

$†$ Presented to the 6th China-Korea International Conference on Multi-functional Materials and Application, 22-24 November 2012, Daejeon, Korea 
influence of different gases such as oxygen, argon, hydrogen and deuterium plasma treatment on the biological properties of polyetheretherketone. The results showed that the contact angle of polyetheretherketone treated with oxygen is obviously smaller that that of polyetheretherketone treated with argon. The deeper the treatment the longer the time required to recover the original hydrophobic character. Furthermore, the hydrogen treatment produced the deepest modification and the oxygen treatment produces a distinctly different modification to that produced by the other gases. On the other hand, Briem et al. ${ }^{6}$ analyzed the influence of plasma treatment environment of polyetheretherketone surfaces on the cell proliferation and differentiation of primary fibroblasts and osteoblasts. The results revealed that the plasma treatment environment has significant effects on the proliferation of fibroblasts and the proliferation rate can either be stimulated or suppressed. The surface activation of polyetheretherketone by $\mathrm{NH}_{3}$ plasma caused the proliferation rate of human fibroblasts was significantly higher compared to control material. Conversely, the surface passivation of polyetheretherketone by $\mathrm{H}_{2}$ plasma caused a suppression of cell growth compared to the control material by means of the DNA content of the cells. Schroder et $a .^{7}{ }^{7}$ investigated the behaviour of human osteoblasts with respect to cell adhesion and growth on plasma-treated polyetheretherketone and Ti surfaces. The results showed that osteoblasts cells adhere faster and occupy a wider cell area on the plasma-treated compared to untreated surfaces.

Surface grafting: Surface grafting modification is mainly through the material surface grafted with hydrophilic or specific functional groups to improve the surface hydrophilicity and wettability, thus enhancing bioactive performance of the material.

In order to promote cell attachment on polyetheretherketone, Joseph and Schwartz ${ }^{8}$ developed a novel surface bioactive treatment technology by grafting nano-scale adhesion layer such as RGD on polyetheretherketone surface. The result showed that the thickness of the adhesion layer can be controlled by exposure time. Compared with untreated polyetheretherketone, the cell adhesion ability and distribution was obviously improved. Kyomoto et al. ${ }^{9,10}$ graft a highly hydrophilic nano-scale bionic lubrication layer on the surface of polyetheretherketone by photo-induced graft polymerization of 2-methacryloyloxy-ethyl phosphorylcholine (MPC). The poly(MPC)-grafted polyetheretherketone surface exhibited unique characteristics such as high wettability and high lubrication as well as high anti-protein adsorption. The density and thickness of the poly(MPC) (PMPC)-grafted layer were controlled by the photo-irradiation time and monomer concentration during polymerization, respectively. The results showed that both the water wettability and lubricity of the PMPC-grafted polyetheretherketone were considerably lower than those of the untreated polyetheretherketone surface.

Chen et $a l .{ }^{11}$ fabricated hydrophilic layer on the surface of polyetheretherketone by grafting of acrylamide (AAm) through ultraviolet irradiation technology. The results demonstrated a significant improvement of surface hydrophilicity for the PAAm-grafted polyetheretherketone with the increase in irradiation time and monomer concentration.
Surface coating: Surface coating technique is mainly to improve the surface bioactivity of polyetheretherketone composite by deposition of bioactive ceramics such as hydroxyapatite and calcium phosphate.

Pino et al. ${ }^{2}$ fabricated apatite layers on the polyetheretherketone surface through exposure to supersaturated simulated body fluid. The results showed that the apatite layer on the surface of polyetheretherketone contained phosphate and carbonate ions, in similar ratios to those in the apatite in dentine, verifying bioactivity improvement of polyetheretherketone after surface coating apatite layer. Han et al. ${ }^{12}$ prepared polyetheretherketone coated with a pure titanium layer using an electron beam deposition method in order to enhance its biocompatibility and adhesion to bone tissue. The results showed that the surface of polyetheretherketone formed a dense, uniform and well crystallized Ti layer without deteriorating the characteristics of the polyetheretherketone implant. The Ti coating layer strongly adhered to the substrate and remarkably enhanced its wettability. Both the in vitro cellular behaviours and in vivo osteointegration experiments showed that the proliferation and differentiation level of cells doubled after the Ti coating. Furthermore, the in vivo animal tests showed that the Ti-coated polyetheretherketone implants had a much higher bone-in-contact ratio than the pure polyetheretherketone implants. These in vitro and in vivo results suggested that polyetheretherketone coating with Ti significantly improved used of polyetheretherketone for hard tissue applications ${ }^{12}$.

Wang et al. ${ }^{13}$ fabricated polyetheretherketone coated with diamond-like carbon by plasma immersion ion implantation and deposition to enhance the surface properties. The results show that osteoblast attachment, proliferation and differentiation are better on diamond-like carbon/polyetheretherketone than polyetheretherketone. diamond-like carbon/polyetheretherketone combines the advantages of diamond-like carbon and polyetheretherketone and is more suitable for bone or cartilage replacements.

Bioactive polyetheretherketone composites: As an orthopedic implant, excellent bioactivity could effectively improve interfacial bonding strength between implant and natural bone, thus reinforcing the fixation stability of implant with natural bone. In order to enhance the bioactivity of polyetheretherketone biomaterials, addition bio-ceramics such as hydroxyapatite and calcium phosphate into polyetheretherketone is currently used widely.

Bioactive polyetheretherketone composites were created by compounding the polyetheretherketone with calcium phosphate and hydroxyapatite. Tan et al. ${ }^{3}$ fabricated porous hydroxyapatite/polyetheretherketone composite by rapid prototyping techniques. The surface bioactivity of hydroxyapatite/polyetheretherketone was investigated by immersion of specimens in simulated body fluid and in vitro cell culture on the surface of specimens. Both the experiments for the bioactivity evaluation of the hydroxyapatite/polyetheretherketone specimens showed a positive effect ${ }^{3}$. Wilmowsky et al..$^{14,15}$ investigated the effects of laser sintered polyetheretherketone with incorporated osteoconductive and bioactive bone substitution materials such as bioactive glass and $\beta$-TCP on cell 
viability, cell proliferation and cell morphology. The results showed that osteoblasts could proliferate on all samples. Furthermore, the bioactive glass reinforced polyetheretherketone samples showed highest values of osteoblasts proliferation and lowest values for $\beta$-TCP containing samples. The experiment in porcine bone defects verified the excellent bioactivity of laser sintered bioglass reinforced polyetheretherketone biocomposites.

Wong et $a l .{ }^{16}$ developed strontium-containing hydroxyapatite/polyetheretherketone (strontium-hydroxyapatite/ polyetheretherketone) composites as alternative materials for load-bearing orthopaedic applications. Both the apatite formation in simulated body fluid and human osteoblasts-like cell proliferation experiments verified that strontium-hydroxyapatite/polyetheretherketone composite was superior to hydroxyapatite/polyetheretherketone in providing bioactivity. This result was mainly attributed to the existence of strontium, in the form of strontium-containing hydroxyapatite (Sr-HA), was confirmed to enhance bioactivity in the polyetheretherketone composites.

\section{Conclusion}

Polyetheretherketone has shown excellent biocompatibility and was widely used in several orthopaedic applications, including the femoral component of total hip replacements and spinal implants. However, the bioactivity of polyetheretherketone would be further improved while it is used as orthopaedic implants. Recently, there have been many different modification technologies to improve the biological performance of polyetheretherketone, especially for the improvement of bioactive properties. Addition of bio-ceramics such as hydroxyapatite and TCP particles into polyetheretherketone matrix is widely applied to improve bioactive performance of polyetheretherketone. However, hydroxyapatite/polyetheretherketone and $\mathrm{TCP} /$ polyetheretherketone composites have involved a trade-off in mechanical properties in exchange for their increased bioactivity. Therefore, how to effectively improve bioactivity of polyetheretherketone under the premise of retaining the mechanical properties is an important research direction.

\section{ACKNOWLEDGEMENTS}

This work was supported by the National Natural Science Foundation of China (Project No. 51175004).

\section{REFERENCES}

1. A. Corvell, J.C. Roberts, P.J. Biermann and J.H. Cranmer, J. Mater. Sci., 34, 2421 (1999).

2. M. Pino, W. Chrzanowski, D. Fabel, M. Baklar, N. Stingelin and K.E. Tanner, Adv. Eng. Mater., 12, B234 (2010).

3. K.H. Tan, C.K. Chua, K.F. Leong, M.W. Naing and C.M. Cheah, Proc. IMechE. Part H: J. Eng. Med., 219, 183 (2005).

4. S.W. Ha, M. Kirch, F. Birchler, K.L. Echert, J. Mayer, E. Wintermantel, C. Sittiq, K.I. Pfund, M. Textor, N.D. Spencer, M. Guecheva and H. Vonmont, J. Mater. Sci.: Mater. in Med., 8, 683 (1997).

5. D.R. Mckenzie, M.K. Newton, P. Ruch, M.M. Bilek and B.K. Gan, Surf. Coat. Technol., 186, 239 (2004).

6. D. Briem, S. Strametz, K. Schroder, N.M. Meenen, W. Lehmann, W. Linhart, A. Ohl and J.M. Rueger, J. Mater. Sci.: Mater. in Med., 16, 671 (2005)

7. K. Schroder, B. Finke, H. Jesswein, F. Luthen, A. Diener, R. Ihrke, A. Ohl, K.D. Weltmann, J. Rychly and J.B. Nebe, J. Adhesion Sci. Technol., 24, 905 (2010).

8. T.J. Dennes and J. Schwartz, J. Am. Chem. Soc., 131, 3456 (2009).

9. M. Kyomoto, T. Moro, Y. Takatori, H. Kawaguchi, K. Nakamura and K. Ishihara, Biomaterials, 31, 1017 (2010).

10. M. Kyomoto and K. Ishihara, ACS Appl. Mater. Interf., 1, 537 (2009).

11. R.C. Chen, H. Sun, A. Li and G.Z. Xu, Chem. Res. Chinese Univ., 28, 162 (2012).

12. A.M. Han, E.J. Lee, H.E. Kim, Y.H. Koh, K.N. Kim, Y. Ha and S.U. Kuh, Biomaterials, 31, 3465 (2010).

13. H.Y. Wang, M. Xu, W. Zhang, D.T.K. Kwok, J. Jiang, Z.W. Wu and P.K. Chu, Biomaterials, 31, 8181 (2010).

14. A.V. Wilmowsky, E. Vairaktaris, D. Pohle, T. Rechtenwald, R. Lutz, H. Munstedt, G. Koller, M. Schmidt, F.W. Neukam, K.A. Schleqel and E. Nkenke, J. Biomed. Mater. Res.-Part A., 87, 896 (2008).

15. V. Wilmowsky, R. Lutz, U. Meisel, S. Srour, S. Rupprecht, T. Takeshi, E. Nkenke, K.A. Schleqel, D. Pohle, H. Munstedt, T. Rechtenwald and M. Schmidt, J. Bioact. Compat. Polym., 24, 169 (2009).

16. K.L. Wong, C.T. Wong, W.C. Liu, H.B. Pan, M.K. Fong, W.M. Cheung, W.M. Tang, K.Y. Chiu, K.D. K. Luk and W.W. Lu, Biomaterials, 30, 3810 (2009). 